\title{
Initial Design and Features of an Augmented Reality System for Urban Park Touring and Management
}

\author{
Konstantinos P. Ferentinos, Yannis Stavrakas, Harry Nakos, Kostis Pristouris, and Myrto S. Barda
}

\begin{abstract}
Urban parks and open green areas are important attractions of environmental interest to city residents and visitors. Careful and well-targeted promotion of these areas, not only enhances the importance of their existence in the urban space, but at the same time can assist in the development of alternative forms of "green tourism", and towards the direction of environmental awareness among citizens, which is particularly important nowadays and crucial for the future of the planet. New technologies are a key tool in enhancing the experience of touring urban parks, as they can make the tour much more attractive, highlighting interesting information about the flora and fauna of the park, as well as various other points of interest. They can also assist park managers in organizing events, thus solving one of the key operating problems mainly of large-scale urban parks, which is the failure to exploit their entire site due to reduced or problematic accessibility. This paper presents the initial design methodology and the main features of an integrated system that comprises an augmented reality mobile application for visitors of urban parks, and a corresponding park management web application for the managers of such park. Through the mobile app, an attractive, interactive touring environment will be created which will highlight the environmental and historical interest of those sites. At the same time, the web application will receive multimedia data from the users and will automatically collect anonymous data that may be useful to park managers to improve the visitors' touring experience.
\end{abstract}

Index Terms-Augmented reality, mobile application, urban parks, web application.

\section{INTRODUCTION}

There are thousands of urban parks around the world, with millions of daily visitors. In addition to the environmental benefits that these parks provide to their hosting cities, they also constitute recreational spaces for citizens and city visitors. However, in most cases, their attractiveness could be further increased and could be focused to specific target groups that could benefit from what these parks have to offer. At the same time, given the existence of appropriate technological tools, park administrators could also benefit from information provided by the visitors themselves or statistical analysis of automatically generated information from visitors' behavior during touring in the parks.

Manuscript received May 6, 2020; revised July 27, 2020. This work has been co-financed by the European Union and Greek national funds through the Operational Program Competitiveness, Entrepreneurship and Innovation, under the call RESEARCH - CREATE - INNOVATE (Project "VR-Park", code: T1EDK-02182).

K. P. Ferentinos and M. S. Barda are with the Dept. of Agricultural Engineering, Soil \& Water Resources Institute, Hellenic Agricultural Organization “Demeter”, Athens, Greece (e-mail: k.ferentinos@swri.gr).

Y. Stavrakas, H. Nakos, and K. Pristouris are with the Information Management Systems Institute, "ATHENA" Research and Innovation Center, Athens, Greece.
Thus, new technologies can definitely be used to make the touring of urban parks not only more attractive and useful, but also safer and more rewarding to the visitor [1], [2]. At the same time, if used properly, they could offer very useful information to park administrators, regarding several aspects of park management and touring services, such as: a) visitors demographics, areas/paths popularity and other touring related information, b) problem reporting and relevant feedback from visitors, and c) interaction with visitors concerning event and/or alert announcements, etc.

However, the majority of current research focuses primarily on computer vision and tracking, or investigates the needs of urban residents, who are already familiar with their environment [3]. This paper proposes the development of an augmented reality (AR) system for urban park touring and management, which adopts a user-centered approach into the design, focusing on an empirical methodology for the investigation and analysis of the requirements of uses who are not familiar with the park environment, while at the same time, park managers take advantage of all types of user activity information to improve park operation. Thus, in this paper, the initial design concepts of the proposed system are presented. In Section II, existing relevant systems are explored. Section III presents the basic methodology for system requirements for both types of users: park visitors and park managers. Then, in Section IV the system design and main features are presented, while Section V analyzes some foreseen operational problems and possible solutions. Finally, Section VI concludes the paper with some future directions towards system implementation and validation.

\section{EXISTING URBAN PARK TOURING APPLICATIONS}

In the field of urban park touring, existing AR applications are primarily aimed at facilitating visitors' touring inside the parks, promoting specific points of interest, and improving the experience of sightseeing in the park area. The vast majority of them (e.g., REI National Park Guide \& Maps [4], Tuscany+ [5], Krka National Park [6], Chimani Viewport Tool [7], and others) focus their AR features towards simple touring facilitation and the provision of information for specific points of interest (POIs), while very few of them also provide information about plants and trees of the park (e.g., Central Park Entire [8]) or allow the users to report specific problems in the park (e.g., Park Path ${ }^{\mathrm{TM}}$ - Local Park Finder [9]).

Table I presents the frequency of occurrence of the most important features among 12 of the most successful AR applications for urban park touring. It is clear from the presented results that navigating visitors to the park and locating the park's points of interest are the main services that 
these applications offer, as these features are provided by all 12 considered applications. Equally important is the availability of the application in English, although for many of these applications English is their basic language. The additional provision of information and historical data for various parts of the parks is also a service provided by most existing applications (75\% of them). However, when the services contain elements of AR and artificial intelligence, like a) showing the distance to specific points of interest through the camera, b) using the camera of the device to navigate the park, and c) identifying plant species, availability percentages drop considerably $(33 \%, 33 \%$, and $17 \%$, respectively). Finally, the very important feature of problem reporting using relevant multimedia material, is only available in one of the 12 applications under review.

TABLE I: FREQUENCY OF OCCURRENCE OF THE MOST IMPORTANT

FEATURES AMONG 12 EXISTING AR APPLICATIONS FOR URBAN PARK TOURING

\begin{tabular}{ll}
\hline \hline Feature & Percentage of occurrence (\%) \\
\hline User navigation inside the park & 100.0 \\
$\begin{array}{l}\text { Localization of Points of Interest } \\
\text { (POIs) }\end{array}$ & 100.0 \\
Availability in English & 100.0 \\
Provision of historical information & 75.0 \\
User's distance from POIs using AR & 33.3 \\
Camera use capability for navigation & 33.3 \\
Plant species identification & 16.5 \\
Problem reporting with image data & 8.3 \\
\hline \hline
\end{tabular}

The main advantages of the proposed system for urban park touring and management against existing applications, are the following:

- Its rich database of information on entities of all types that exist in an urban park. The user will be receiving information through AR about specific trees and plants on his/her field of view, as well as other POIs that may be found in the surrounding area (e.g., statues, monuments, buildings of special interest, etc.).

- The interactivity in the user interface. The user of the mobile application of the proposed system will not only be the recipient of rich information, but also a transmitter of such information. User involvement will not be limited to social media but also to reporting problems, sending comments and transmitting multimedia material to the park administrators.

- The provision of digital services to stakeholders. Park administrators that will use the management application of the proposed system, will have the ability to be informed of any problems in the park area, as well as to receive useful usage statistics, such as visitors' routes and gathering points or locations, with the goal of continuously improving the visitor's touring experience.

\section{USER REQUIREMENT ANALYSIS METHODOLOGY}

The development of user requirements of an information system is a process which includes the contribution of both the systems analyst and the end-users, since the specific requirements come as a result of potential system use scenarios that have to cover the widest use range possible.
Usage scenarios are in direct association with interest groups. Interest groups are groups of users who use the system in different ways, for different reasons and for different purposes. Therefore, the system should provide functions and capabilities tailored to each interest group. In the proposed system, the main interest groups are two: park visitors and park managers. Each interest group (especially visitors) can contain of course, many different user categories.

By creating the baseline usage scenarios, possible use-cases for each usage scenario can be described. These use-cases refer to specific application usage situations, which create different user needs from the system. Therefore, these use-cases will result in the different user requirements that need to be implemented in system development. This is the basic methodology of extracting and analyzing the touring requirements of the system to be developed. The following sub-sections give the basic structure and the characteristics of the three basic parameters of this approach, namely the usage scenarios, the use-cases, and finally the touring requirements. Based on the topic of the proposed system, four different usage scenarios (US) can be distinguished:

- US1: Urban park touring

- US2: Input and management of entities of interest

- US3: Two-way communication of the two main interest groups

- US4: Exploitation of park visitors touring data

The first US refers to the most important of the several uses of the application, which concerns the visitors of the park. It includes all the possible actions that park touring might involve, for any type of visitor. The second US concerns the ability of the system administrator to manage the entities of the park and all relevant information in the system database. These entities can be categorized into some basic groups: i) plant material, ii) monuments/statues, iii) urban equipment (benches, lights, etc.), iv) gathering points, v) other POIs. The third US concerns the exchange of information between park visitors and managers. Visitors should be able to send reports on specific problems that they encounter in the park, while managers should be able to send announcements regarding park operations, events, etc., and security notifications. Finally, the fourth US has to do with data that can be collected anonymously by the mobile devices running the application, which concern location and demographic information and can be used by managers to draw specific conclusions on the touring habits of visitors.

For each of these four usage scenarios, a number of specific use-cases have been considered, leading to corresponding user requirements that the proposed system should cover through its included features and operational characteristics. Operational requirements were divided into two basic categories: those that concerned the park visitors, and those that concerned the park managers and system administrators. In addition, some non-operational requirements were considered, concerning system reliability, performance, usefulness, transferability, and security. Finally, technical requirements concerning the necessary hardware components (camera, GPS, etc.) of the mobile and server equipment that will run the corresponding subsystems, were also considered. 


\section{System DESIGN AND MAIN FEATURES}

Each of the four main usage scenarios presented in the previous section (US1-US4), is essentially implemented by a corresponding subsystem of the integrated system, as follows:

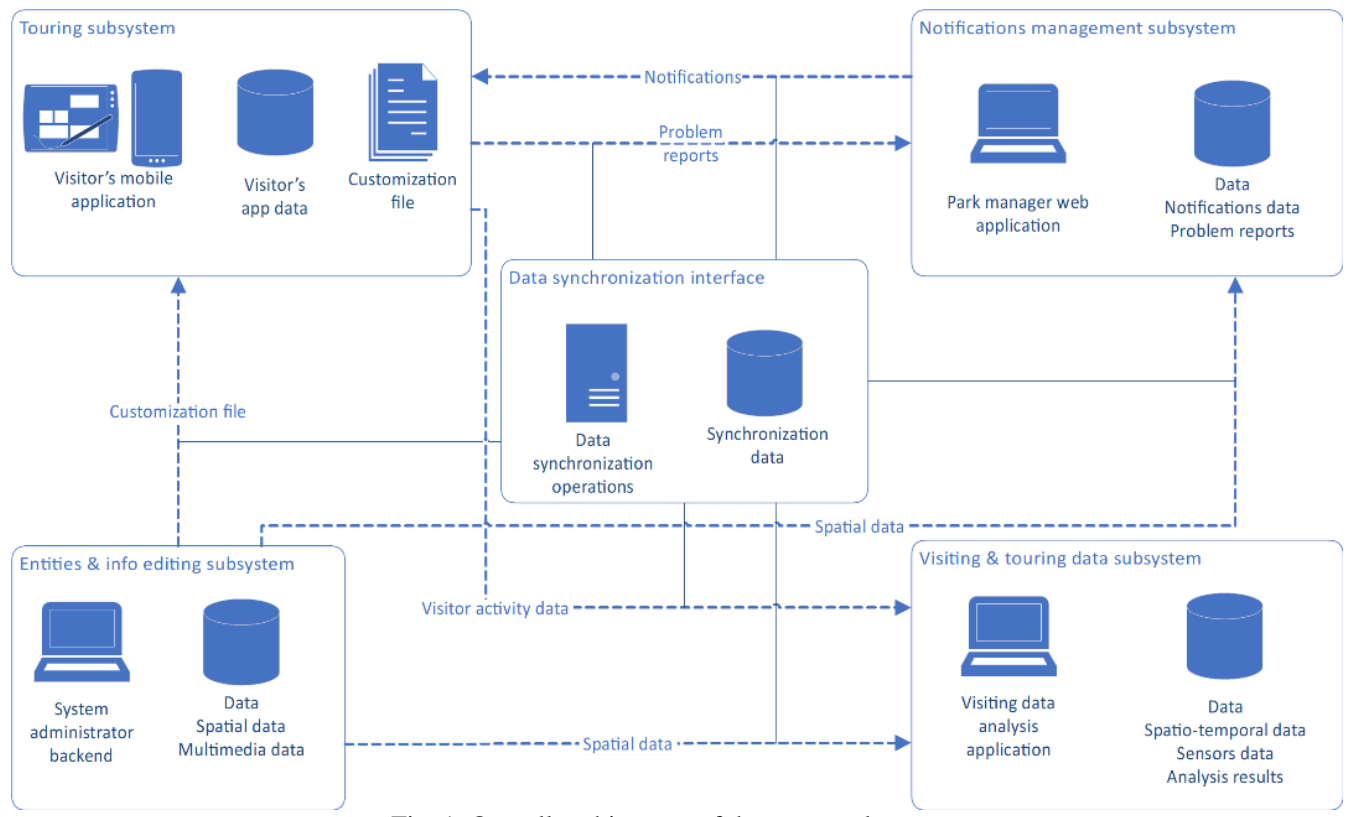

Fig. 1. Overall architecture of the proposed system.

US1: Touring subsystem - This subsystem mainly concerns the visitor's mobile application of the system. This application provides navigation services to the visitors and, through its AR environment, gives additional information on POIs in the park, as well as events and alerts notifications. Thus, the user of the application uses the corresponding subsystem for purposes that have to do with either the location or the corresponding information of the surrounding POIs, information on current events in the park, or to search for interesting paths inside the park. Navigation or information data is dynamic and can change based on the user's movement and the view point of the used mobile device.

US2: Entities and information editor subsystem - This subsystem is operated by the administrator of the proposed system, who can be the park manager. Alternatively, the subsystem can be provided as a service to the park managers' team. Its role is to provide functions for input and management of the entities of interest inside the park, which are incorporated into the visitor's AR application. In addition, the subsystem provides editing capabilities for menus and specific functions of the visitor's mobile app.

US3: Notifications management subsystem - This subsystem deals with the operation of the two-way communication between the two main types of users of the system: park visitors and park managers. The communication concerns two types of messages: problem reports, and announcements / notifications. Park visitors, which use the AR mobile application, can send reports on specific problems that they identify during their park visit, which may concern park operation problems, specific issues on existing entities or equipment, or even security concerns. Park managers, through their web application, can manage these reports, give them priorities, mark their resolution status, etc. The second type of messages managed by the specific subsystem concerns announcements about specific current and future events or operational issues of the park, and possible security alerts or warnings that have to reach the visitors through their mobile apps as soon as possible.

US4: Visiting and touring data subsystem - This subsystem deals with the collection and exploitation of park visiting and touring data. It uses information on the behavior of the park visitors, which collects it anonymously through the mobile application usage, like, for example, common paths and routes, points of gathering, point of increased interest, etc. Park managers can use the subsystem through the web application of the system to analyze visitors touring habits and use the data to improve park exploitation and thus make the touring experience better.

Each of the above subsystems includes the necessary infrastructure and backend or frontend features through which the objectives of the corresponding usage scenarios are materialized. In addition, to complete their individual functions, the subsystems are required to exchange and share data structures between them. In order to communicate as independently and as asynchronously as possible, the architecture incorporates a Data Synchronization Interface, in which the individual communication functions between the subsystems is assembled. These are depicted in Fig. 1.

The following sub-sections present the main design approaches for each of the four main subsystems.

\section{A. Touring Subsystem}

The Touring subsystem will comprise the main part of the $\mathrm{AR}$ application for the visitors of urban parks. It will create an interactive touring environment that highlights the environmental and historical interests of the site. The subsystem will use AR technologies and, based on the location and orientation of the user, will provide real-time information about points of interest (trees, gardens, statues, monuments, etc.) found in the field of view of the user. It will also be possible for the user to select a predetermined route that he/she wants to follow, or to make his/her own route 
based on the points of the park that interest him/her. The system will help the user navigate to the next point of the route at a time. Also, the Touring subsystem will present in real-time information about periodic actions taking place in the park. The content of the subsystem that concerns park entities that do not change frequently, will be dynamically loaded through the "Entities and information editor" subsystem, which will determine what will appear in the application. On the other hand, the dynamic content of the application, which changes on a daily basis, will be loaded through the "Notifications management" subsystem.

The architecture of the Touring subsystem is focused on two basic functions: Touring and Notifications. Its connection to the other subsystems is realized through corresponding APIs. Problem reports registered by the users, feed the Notifications management subsystem. All the material of the Touring subsystem is extracted from the Entities and information editor subsystem. Every time the application opens on the visitor's mobile device, the Touring subsystem queries the corresponding API of the Entities and information editor subsystem whether there is a change in the content, to download it. The same process is performed by the Notifications management subsystem, concerning existing park events and announcements. If the mobile device has no Internet connection (no $\mathrm{WiFi}$ available or data is disabled), there will be a relevant notification to the user. For the disclosure of usage data, the user's consent will be asked during the installation of the application, and the process will be able to be disabled at any time, through the application settings.

\section{B. Entities and Information Editor Subsystem}

The Entities and information editor subsystem will manage all park entities, their categorization, their spatial dimension, the metadata describing them, the digital material that is connected to them (photos, sounds, videos), and any AR signals connected to them. This subsystem will also affect the operating parameters of the visitor's mobile application, as to the type of information to be provided to the user (which entities will be displayed to the user), the level of detail provided, etc. For example, urban equipment and emergency meeting points may not be visible to the visitor unless the park manager wants it. Additionally, this subsystem also creates the routes that are suggested to the visitors.

Park managers, through the specific subsystem, will be able to import new entities into the park, modify existing ones, and delete them. Once they have completed the desired changes, they will create an updated version of the mobile application, which will be downloaded to the mobile devices when the application opens and the devices are connected to the internet. Communication will be performed through OAuth2 [10] for secure access via Client Id and Client Secret [11], and Access and Refresh tokens. In addition, the administrator of the subsystem will be able to add users to the park manager's web application, and assign specific roles to them (e.g., simple park manager or park manager with editing privileges).

The subsystem will have a front-end from where all the content and user management tasks will be performed, and a backend where all processing and storage functions will be run in the database, and where the interface API will run.

\section{Notifications Management Subsystem}

The notifications management subsystem is responsible for two basic functions of the proposed system: (a) sending and managing reports of problems in the park, and (b) sending notifications and any type of announcements to the visitors of the park. Due to the involvement of both park visitors and park managers in this particular subsystem, its design requires the creation of two subsystem subsets, one in the visitor's AR application and the other in the park manager's application. Sending problem reports is performed by users of the visitor mobile application via a simple interface in which:

- The type of problem is selected by the user through a predefined list of basic choices (e.g., Plant material, Building/Construction/Path, Bench, Lighting, Water/Irrigation, Pollution, Danger)

- The camera application of the mobile device is used to attach a relevant photograph to the report

- The GPS of the mobile device is used to establish the location of the reported event

- It is possible for the user to optionally add short comments to the report (problem description).

Problem reporting is received by the corresponding subsystem of the park manager's application. In that, the park manager has the ability to investigate the reports, to group them accordingly, and to set specific resolution priorities. In addition, the manager can register the reports as resolved when all required actions for their resolution have been completed by the park staff. At the same time, visitor users can view their registered problem reports, as well as the reports that have been registered by all users of the application. In addition, they can view specific statistics on the various reports, such as percentages per problem category, average resolution times, etc.

As far as notifications and announcements are concerned, park managers can send push-up notification at any time to all park visitors who run the mobile application, as well as send announcements for various park activities, events or any other information regarding park operation (e.g., change of visiting hours, etc.).

The three main structures of the subsystem are the backend and the two corresponding frontends, one for the visitor's mobile application and one for the park manager's web application. The Data Synchronization Interface, which is integrated into some Central Management System of the entire proposed system, performs all the necessary data flow supervision and synchronization. All data of the subsystem, whether it concerns problem reports or notifications/announcements, is stored in the subsystem database. Whenever a user reports a problem via the corresponding interface of the visitor's application, the data (of the sensors of the device and that generated by the user's selections and inputs, as described above) are sent to the Problem Management module, where the necessary checks are made as to whether the reported problem has already been registered or is a new addition. The data then passes through the Data Pre-processing module to be stored in the subsystem's database. Similarly, if the data concerns a notification or announcement created by the park manager via the corresponding manager application's frontend, this data is sent to a corresponding module that manages these 
notifications, and by following similar steps as before, they are stored in the subsystem database. In the case of data retrieval from both applications (visitor's and park manager's), the two basic modules of Data Analysis and Data Retrieval are used, for both cases (notifications and problem reporting).

\section{Visiting and Touring Data Subsystem}

The Visiting and touring data subsystem allows the park manager to investigate visiting patterns in areas, paths, and specific places in the park. For this purpose, it collects visitor activity data recorded in the Touring Subsystem and then made available through the Data Synchronization Interface. The Visiting and touring data subsystem periodically receives the visitor activity data in the form: <application ID, location, timestamp>, where the application ID is the unique ID of the visitor's application, the location is the exact visitor's GPS location, and the timestamp is the exact time of the positing logging. The subsystem creates similar triplets every 30 seconds (for each visitor) and sends them asynchronously when the mobile device connects to the network. Additionally, the subsystem updates the park spatial data via the "Entities and information editor subsystem", through the Data Synchronization Interface. On all this information, the park manager can perform queries and analyses, the results of which are either displayed on the Interactive Map component of the system or are stored as reports. The subsystem is based on three pillars in order to present the visitors behavior: (a) traffic per park area, (b) traffic per park path, and (c) traffic per point of the park.

\section{E. System Structure and Main AR Features}

The overall structure of the proposed system takes the general form shown in Fig. 2. The component in the middle that connects the two basic applications of the system (Interactive Map Component) serves as a common ground of the two applications, the visitor's AR mobile app and the manager's web app, and provides visualization features and editing features to both basic applications.

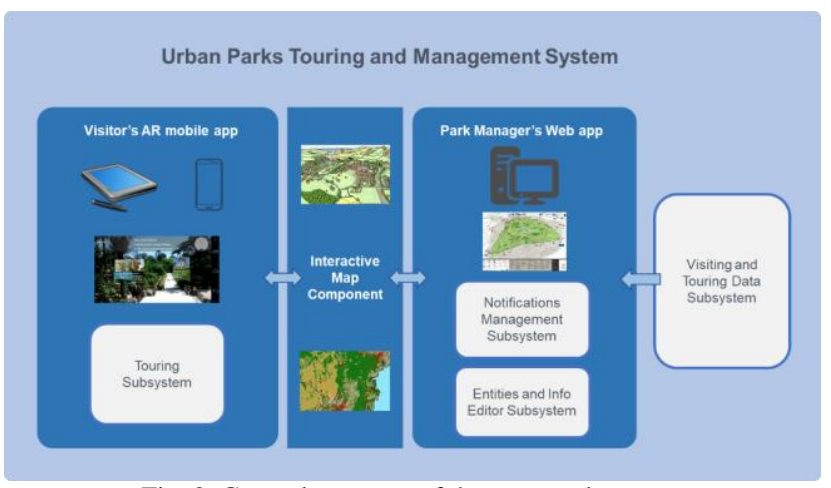

Fig. 2. General structure of the proposed system.

The exact AR user interface is yet to be determined, however, its basic features have already been defined. It will include a "Discover the Park" section with a categorization of park entities (monuments-statues, botanical gardens, shelters, palm trees, other trees and plants of specific interest, etc.). Each category will display its corresponding content. Selecting this content will show detailed information for each entity. There will be also navigation instructions of each entity, and augment content will be provided, if such content exists.

A second section will be called "Recommended Routes". The user will see specific labeled routes, and by selecting them, he/she will be able to see the points that comprise them. By selecting a point, the user will be able to see more information about it. If navigation is selected, the user will be taken to the first point of the route, and so on. The user will also be able to create her own routes ("My Routes"). A name should be entered for the route, together with the choice of specific entities that will be included in the route, which is then stored locally, on the mobile device.

Another option will be the ability to see what is around the user ("Near Me"). Selecting this option will open the camera of the mobile device, and the user will be able to rotate it in all directions and see which park entities are nearby, in the foreground and background of the field of view of the device. Finally, the user will have some additional options like "Favorites", where favorite park entities can be stored, and "Search", where he/she can search for some of the information contained in the application.

\section{DISCUSSION}

AR technologies constitute a very promising approach to making urban park environments and other touristic sites more attractive, especially when combined with gamification methods and other learning techniques [1]-[12]. However, several practical problems can arise during the operation of an AR system in uncontrolled outdoor environments, especially with complex objects like trees and plants that exist in urban parks. A main limitation of such AR systems is that they require a high registration precision [13]. In such outdoor scenarios, GPS and digital compass provide position and orientation data, but their accuracy is not sufficient for a seamless and well-aligned overlay onto the user's view [14]. The main problem arises when the distance between the user and the object of interest becomes so small that the error of the GPS-provided position makes the AR-provided position of the object to appear at different than its actual position on the user's view, or even to disappear, while in reality it is still in front of the user.

A real-time visual tracking system that uses visual cues of buildings in an urban environment for correcting this problem of conventional tracking systems has been proposed in [14]. However, this approach is focused on buildings, which are rather simpler structures than those existing in urban parks, and it also relies on knowledge of CAD models of the buildings. Another interesting approach proposes the use of input from a video tracker that detects natural 2-D features in the video sequence to reduce such errors [15], while more recently, similar but more sophisticated approaches based on deep learning [16] have been developed [17].

In our work, a similar deep learning method will be developed to be integrated into the proposed AR system, focused on urban park entities and based on simple GPS correction rules. An object detection model will be developed and trained to identify the park entities included into the AR application. The detection will be happening in real-time and when the distance between the user and a specific object of interest becomes smaller than a predefined threshold, the 
GPS-provided location information will be overridden and possibly corrected by the output of the object identification and tracking model.

The proposed model will be trained on image data of several urban park entities acquired during several environmental conditions in the system's testing site. It will be based on existing sophisticated object tracking models, such as the recent YOLOv3 [18] which can meet the time-demanding constraints of the proposed application.

\section{CONCLUSIONS}

In this work, the initial design methodology of an AR system for urban park touring and management was presented, and its basic features regarding visitor experience and park management were analyzed. The proposed system comprises of two basic applications, the visitor's AR mobile app, and the park manager's web app. The design of both applications is based on the identification of four basic usage scenarios, which lead to the extraction of specific use cases, leading to the definition of user requirements for the development of the integrated system.

In addition, the main features of the proposed system were described and some foreseen operational problems related to location registration errors were discussed, while the proposed solution based on real-time object identification models and their integration into the AR application was analyzed. The initial version of the system will be tested at "Pedion Areos" urban park [19] in Athens, Greece, and assessed in real operating conditions so that useful insights can be drawn towards its improvement.

\section{CONFLICT OF INTEREST}

The authors declare no conflict of interest.

\section{AUTHOR CONTRIBUTIONS}

KPF and YS conceived the research idea and supervised the research; HN and KP designed the system; MSB performed the literature review. KPF and MSB wrote the paper; all authors have approved the final version.

\section{REFERENCES}

[1] L. Pombo, M. M. Marques, V. Carlos, C. Guerra, M. Lucas, and M. J. Loureiro, "Augmented reality and mobile learning in a smart urban park: pupils' perceptions of the EduPARK game," in Citizen, Territory and Technologies: Smart Learning Contexts and Practices, Ó. Mealha, M. Divitini, and M. Rehm, Eds. Springer, Cham., 2018.

[2] M. C. Dieck and T. Jung, "A theoretical model of mobile augmented reality acceptance in urban heritage tourism," Current Issues in Tourism, vol. 21, pp. 154-174, 2018.

[3] Z. Yovcheva, D. Buhalis, C. Gatzidis, and C. P. Elzakker, "Empirical evaluation of smartphone augmented reality browsers in an urban tourism destination context," International Journal of Mobile Human Computer Interaction, vol. 6, pp. 10-31, 2014

[4] REI Mobile Apps, always ready for your next adventure. [Online]. Available: https://www.rei.com/mobile

[5] Augment your journey, Tuscany+. (2018). [Online]. Available: http:// augmentyourjourney.altervista.org/en-tuscany/

[6] Augmented Reality of Krka National Park Available via a New Mobile $\begin{array}{lll}\text { Application. (2016). } & \text { [Online]. Available: }\end{array}$ http://www.npkrka.hr/clanci/AR-Krka-National-Park-Tour\%20pro\% C5\% A1irena\%20-stvarnost/262/en.html

[7] Using Chimani's Viewport Tool to Navigate Bryce Canyon National Park. [Online]. Available: https://blog.chimani.com/2016/06/28/using-chimanis-viewport-tool-to -navigate-bryce- canyon-national-park

[8] Central Park Entire. [Online] http://www.centralparkentireapp.com /about.html

[9] Park Path App. [Online]. https://www.nrpa.org/our-work/park-path-app

[10] OAuth 2.0. [Online]. Available: https://oauth.net/2/

[11] The Client ID and Secret. [Online]. Available: https://www.oauth.com/oauth2-servers/client-registration/client-id-sec ret/

[12] Z. Yovcheva, D. Buhalis, and C. Gatzidis, "Smartphone augmented reality applications for tourism," E-Review of Tourism Research, vol. 10, pp. 63-66, 2012

[13] P. Pace, G. Aloi, and A. Palmacci, "A multi-technology location-aware wireless system for interactive fruition of multimedia contents," IEEE Transactions on Consumer Electronics, vol. 55, pp. 342-350, 2009.

[14] R. Behringer, J. Park, and V. Sundareswaran, "Model-based visual tracking for outdoor augmented reality applications," in Proc. the International Symposium on Mixed and Augmented Reality, 2002, pp. 277-322.

[15] R. Azuma, J. W. Lee, B. Jiang, J. Park, S. You, and U. Neumann, "Tracking in unprepared environments for augmented reality systems," Computers \& Graphics, vol. 23, pp. 787-793, 1999.

[16] Y. LeCun, Y. Bengio, and G. Hinton, "Deep learning," Nature, vil. 521, pp. 436-444, 2015.

[17] J. Rao, Y. Qiao, F. Ren, J. Wang, and Q. Du, "A mobile outdoor augmented reality method combining deep learning object detection and spatial relationships for geovisualization," Sensors, vol. 17, 2017.

[18] J. Redmon and A. Farhadi, "YOLOv3: An incremental improvement," arXiv preprint 1804.02767, 2018

[19] Pedion Areos - Athens, Attica, Greece. (2019). [Online]. Available: https://athensattica.com /things-to-do/parks/pedion-areos

Copyright $\odot 2020$ by the authors. This is an open access article distributed under the Creative Commons Attribution License which permits unrestricted use, distribution, and reproduction in any medium, provided the original work is properly cited (CC BY 4.0).

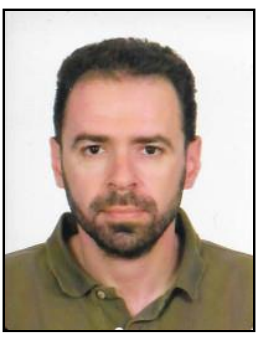

Engineering)
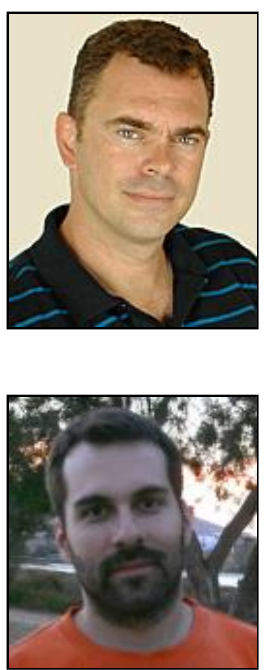

Konstantinos P. Ferentinos received his BSc degree in agricultural engineering from the Agricultural University of Athens, Greece, in 1997, and his MS and $\mathrm{PhD}$ degrees in biological and environmental engineering with a minor in computer science (artificial intelligence) from Cornell University, Ithaca, NY, USA, in 1999 and 2002 , respectively.

$\mathrm{He}$ is currently a researcher at the Hellenic Agricultural Organization "Demeter" (Institute of Soil \& Water Resources, Dept. of Agricultural

Yannis Stavrakas received his diploma in physics from the University of Athens, his MSc in computer science from University College London, and his $\mathrm{PhD}$ in informatics from the National Technical University of Athens.

Since 2007 he is a researcher at the Information Management Systems Institute of the Research Center "Athena". He has participated as a scientific coordinator, technical manager, and developer in many researches, commercial, and consulting projects.

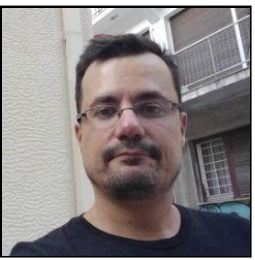

Harry Nakos is an electrical and computer engineering from the National Technical University of Athens. He works as a scientific associate at the Athena Research Center in Athens.

His professional and research interests include web application development, geospatial data analysis, and social network data analysis.

Kostis Pristouris is an experienced software engineer with a diploma in electrical \& computer engineering and a postgraduate diploma in geoinformatics, both from the National Technical University of Athens.

$\mathrm{He}$ is a full stack engineer, with strong experience in web development and databases, particularly versed in geographic information systems applications and infrastructure. 


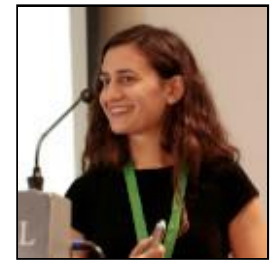

Myrto S. Barda is a research fellow at the Hellenic Agricultural Organization "Demeter" and a $\mathrm{PhD}$ candidate at the Agricultural University of Athens (AUA), Dept. of Crop Science.

She received her BSc from the Dept. of Crop Science of AUA (ranked $1^{\text {st }}$ among all department graduates), and her MSc from the same department, in collaboration with the Imperial College London, Centre for Environmental Policy. 